\title{
BOTULISMO SIMULTÁNEO EN DOS HERMANOS COCAINÓMANOS
}

\section{SIMULTANEOUS BOTULISM IN TWO BROTHERS ADDICTED TO COCAINE}

\author{
AGUIRRE-BALSALOBRE $\mathrm{F}^{1}$, GONZÁLEZ-HERRERA $\mathrm{M}^{1}$, MENGUAL-VERDÚ E², \\ GARCÍA-CONCA V ${ }^{1}$, HUESO-ABANCENS JR ${ }^{2}$
}

\section{RESUMEN}

Introducción: El botulismo es poco frecuente en la actualidad. La toxina botulínica produce una parálisis muscular progresiva que puede producir la muerte del paciente por parada de los músculos respiratorios.

Caso clínico: Dos pacientes hermanos, adictos a la cocaína, acudieron al Servicio de Urgencias por disminución de la agudeza visual cercana, con midriasis bilateral. A los dos días presentaban, además, ptosis palpebral, disfunción asimétrica de la motilidad ocular extrínseca, y vómito.

Discusión: Ante la presencia de una parálisis de la acomodación con una midriasis bilateral que responde a pilocarpina, se debe considerar el botulismo como una posible etiología.

Palabras clave: Botulismo, alteraciones pupilares, reflejos pupilares, abuso de cocaína, midriasis.

\begin{abstract}
Introduction: Botulism is currently an uncommon disease in which the botulinum toxin causes a progressive muscular paralysis that can lead to the death due to a failure of respiratory muscles.

Clinical case: Two brothers, both addicted to cocaine, came to the casualty department because of a decrease of near visual acuity and bilateral mydriasis. Two days later, they developed eyelid ptosis, asymmetric dysfunction of the extraocular muscles and vomiting.

Discussion: The presence of a paralysis of accommodation, with bilateral mydriasis that reacts to pilocarpine, makes it necessary to consider botulism as a possible cause (Arch Soc Esp Oftalmol 2007; 82: 583-586).
\end{abstract}

Key words: Botulism, pupil disorders, pupillary reflex, cocaine abuse, mydriasis.

\footnotetext{
Recibido: 31/5/06. Aceptado: 25/7/07.

Hospital Universitario San Juan de Alicante. Alicante. España.

1 Licenciado en Medicina.

2 Doctor en Medicina.

Correspondencia:

Fernando Aguirre Balsalobre

Servicio de Oftalmología

Hospital Universitario San Juan de Alicante

Ctra. Ncnal. 332 Alicante-Valencia, s/n

Apartado de correos, 41

03550 San Juan de Alicante (Alicante)

España

E-mail: faguir88@hotmail.com
} 


\section{INTRODUCCIÓN}

El botulismo es una enfermedad neuroparalítica causada por las exotoxinas del Clostridium botulinum. Es poco frecuente, y en España se diagnostican entre unos 10 y 50 casos al año (1).

Los modos habituales de contraer la enfermedad en los pacientes adultos son la intoxicación alimentaria, generalmente provocada por la ingesta de conservas caseras sin cocción previa y fabricadas al menos ocho días antes, o bien las heridas cutáneas penetrantes.

Según la cantidad entrada en el organismo, los primeros síntomas se manifiestan desde doce horas hasta ocho días después. Esta toxina produce un bloqueo de la contracción muscular por el bloqueo de la liberación de acetilcolina preganglionar en la placa neuromuscular, hecho que provoca en un primer momento alteraciones oculares como midriasis arreactiva, ptosis palpebral, o diplopía por parálisis oculomotora. A continuación, la debilidad debida a las parálisis musculares se generaliza hasta que se produce una parada respiratoria y el fallecimiento del sujeto $(1,2)$.

El diagnóstico se efectúa por la detección de la toxina en suero u otros fluidos corporales. El tratamiento consistirá en procurar la eliminación de la toxina del tubo digestivo, la administración de un suero antitóxico, penicilina $\mathrm{G}$ y soporte respiratorio. Los síntomas revertirán en orden inverso al que se han producido $(1,2)$.

\section{CASO CLÍNICO}

Dos hermanos de 38 y 42 años de edad acudieron al Servicio de Urgencias por visión borrosa de 24 horas de evolución, peor en visión cercana. Consumían de forma habitual cocaína, y relacionaban la sintomatología actual con ella, ya que se daba la circunstancia de que el día anterior habían cambiado de proveedor.

En ambos casos, la agudeza visual lejana estaba conservada $(0,8)$, pero la agudeza visual cercana era inferior a 0,4. Presentaban una midriasis arreactiva bilateral (figs. 1 y 2) que tras instilar pilocarpina al $1 \%$ regresó en pocos minutos. Los movimientos oculares extrínsecos y la convergencia, así como el resto de la exploración, fueron normales.

Se citaron para reevaluación, diagnosticados de posible midriasis por adulteración de la droga. A los dos días se añadió ptosis palpebral bilateral y diplopía. Existía limitación de la abducción de ambos ojos, estando el resto de posiciones cardinales de la mirada sólo ligeramente restringidas. Además, manifestaban náuseas, vómitos, disfagia, y dificultad miccional.

Profundizando en la anamnesis, comentaron que dos días antes del inicio de los síntomas habían consumido cardos en conserva preparados de forma artesanal. Se planteó la posibilidad de una intoxicación por Clostridium botulinum.

Las pruebas de imagen craneal que se realizaron (tomografía axial computerizada) resultaron ser normales. No así los electromiogramas, que mostraron un bloqueo a nivel de la placa motora muscular, por lo que se inició la administración de antitoxina botulínica.

El menor de los hermanos requirió atención intrahospitalaria durante dieciocho días, y el mayor veintinueve, dado que precisó ventilación mecánica. La detección de la toxina en heces fue negativa en ambos.

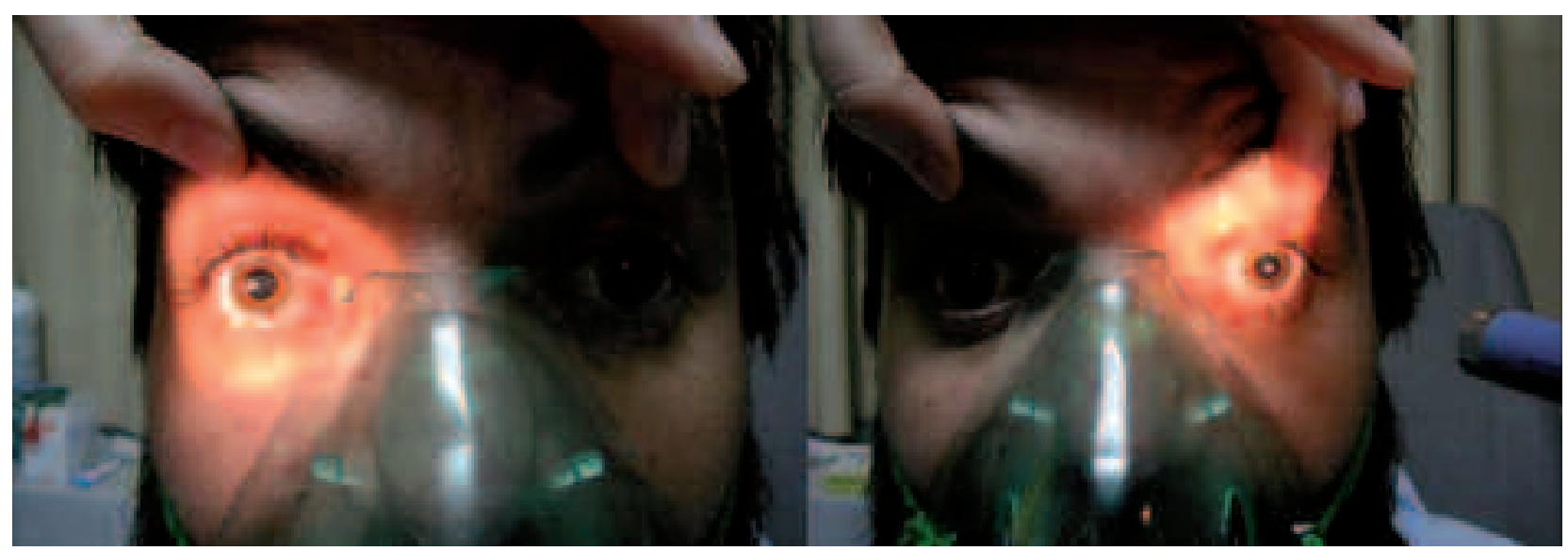

Fig. 1: Midriasis bilateral en el hermano mayor, el más grave, a las tres semanas del inicio de los síntomas. 


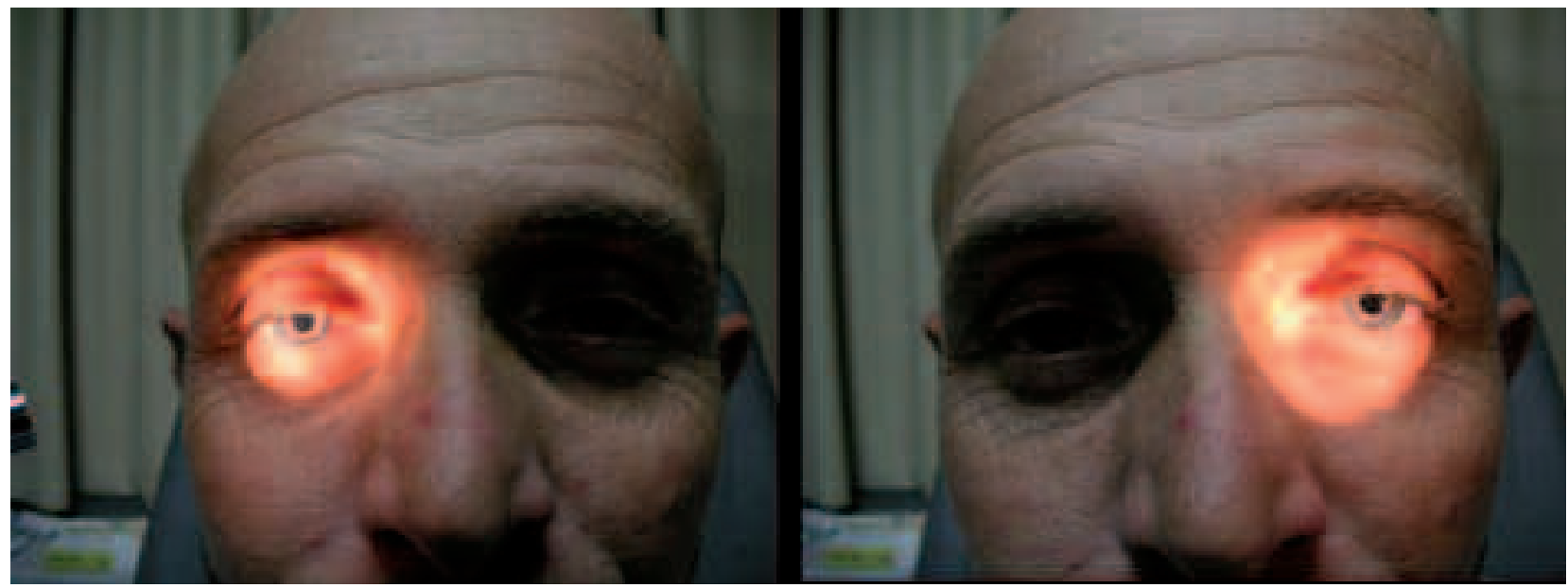

Fig. 2: Midriasis en el hermano menor a las tres semanas del inicio de los síntomas.

La patología ocular remitió totalmente y de forma inversa a como se había producido. La midriasis no desapareció completamente pasados cuarenta días desde su comienzo (figs. 1 y 2), y la mejoría fue algo más temprana en el hermano menor, que padeció el cuadro menos grave (figs. 3 y 4).

\section{DISCUSIÓN}

Ante una midriasis pupilar fija sin traumatismo previo se debe descartar en primer lugar como hecho más frecuente la dilatación pupilar por colirios o productos vegetales, ya sea de forma accidental o voluntaria. También es importante evaluar los movimientos oculares extrínsecos y palpebrales por si la causa fuese una parálisis del III nervio craneal. Si nos encontramos a la biomicroscopía con una pupila irregular en el contexto de una midriasis lentamente reactiva a la luz, la pupila de Adie sería un diagnóstico probable (3).

La instilación de pilocarpina tópica facilita el diagnóstico. Una pupila en la que se ha instilado un parasimpaticolítico, no responde o responde poco a la pilocarpina al $1 \%$. La pupila de Adie se contrae a dosis bajas de pilocarpina $0,125 \%$, mientras que una parálisis del III nervio lo suele hacer a dosis más altas. Estas dos últimas patologías son unilaterales con más frecuencia que la midriasis farmacológica (3).

La acomodación debe ser explorada. Una midriasis bilateral no se debe atribuir a cocaína (estimulación simpática) si existe trastorno de la acomoda-

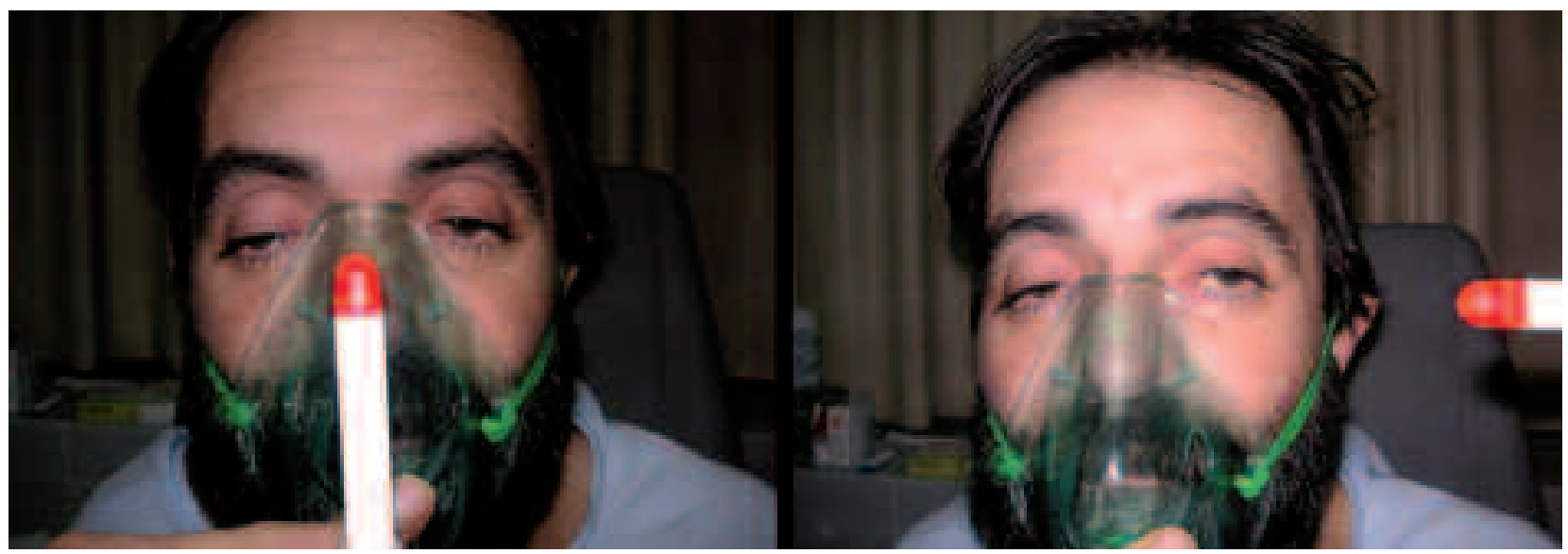

Fig. 3: Ptosis palpebral y debilidad de movimientos oculares en el hermano mayor a las tres semanas del inicio de los sintomas. 


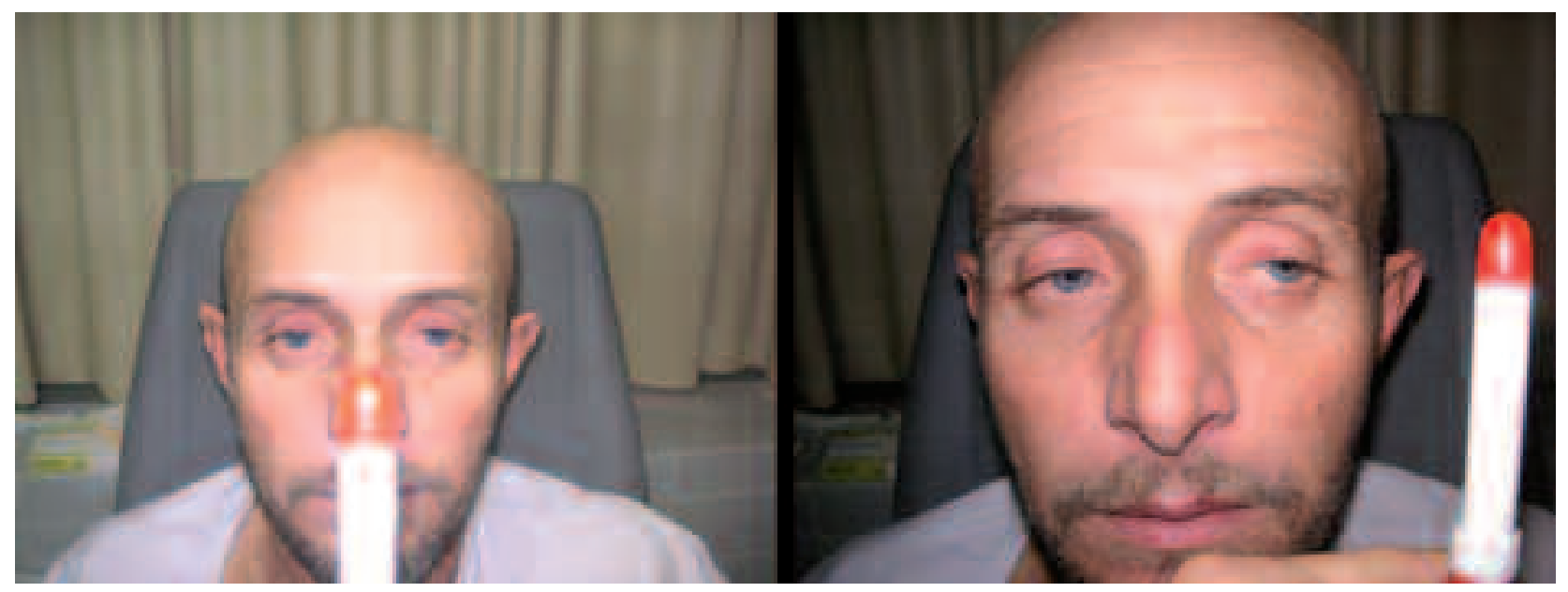

Fig. 4: Menor grado de ptosis y menor limitación de los movimientos oculares en el hermano menor a las tres semanas del inicio de los síntomas.

ción, ya que esto lo provocan los bloqueos parasimpáticos $(4,5)$.

Aunque el botulismo es muy poco frecuente, ante una midriasis bilateral que responde a pilocarpina debemos interrogar al paciente sobre posibles antecedentes alimentarios de interés. Si existen sospechas no deberíamos dudar en remitir al paciente para un estudio electromiográfico. Se debe administrar precozmente la antitoxina botulínica, aun sin confirmar la presencia de toxina en el organismo o en los alimentos, ya que ello no siempre es posible.

En conclusión, el diagnóstico precoz de esta patología es fundamental para el pronóstico vital de los pacientes, de ahí la importancia de tenerla en cuenta en el diagnóstico diferencial de las midriasis bilaterales.

\section{BIBLIOGRAFÍA}

1. Torrabadella de Reynoso P, Piédrola Angulo G, Prat Aymerich C, Vallés Casanova X. Infecciones causadas por bacterias anaerobias esporuladas. In: Farreras, Rozman. Medicina Interna. 14th Ed. Madrid: Harcourt; 2000; II: 2632-2640.

2. Caya JG. Clostridium botulinum and the ophthalmologist: a review of botulism, including biological warfare ramifications of botulinum toxin. Surv Ophthalmol 2001; 46: 25-34.

3. Savino PJ, Sergott RC. Neurooftalmología. In: Rhee, Pyfer. Manual de Urgencias Oftalmológicas. 3rd Ed. Philadelphia: Mc Graw-Hill Interamericana; 2001; 301-364.

4. Penas SC, Faria OM, Serrao R, Capao-Filipe JA, MotaMiranda A, Falcao-Reis F. Ophthalmic Manifestations in 18 patients with botulism diagnosed in Porto, Portugal between 1998 and 2003. J Neuroophthalmol 2005; 25: 262-267.

5. Simcock PR, Kelleher S, Dunne JA. Neuro-ophthalmic findings in botulism type B. Eye 1994; 8: 646-648. 